\title{
ADAPTAÇÃO ACADÊMICA E SAÚDE MENTAL DE ESTUDANTES DE MEDICINA NA COVID19: ESTUDO EXPLORATÓRIO NO BRASIL
}

\author{
Gabriela Tacaci Michelis ${ }^{1}$, Julia Galvão², Samara Almeida de Freitas ${ }^{3}$, Camélia Santina Murgo ${ }^{4}$, Celeste Corral \\ Tacaci Neves Baptista ${ }^{5}$
}

${ }^{1}$ Graduanda da Faculdade de Medicina na Universidade do Oeste Paulista -UNOESTE. Graduada em Ciências Biológicas pela Universidade de Brasília - UnB. ORCID iD: https://orcid.org/0000-0002-9524-5662. E-mail: gabi michelis@hotmail.com

${ }^{2}$ Graduanda da Faculdade de Medicina na Universidade de Taubaté - UNITAU. ORCID iD: https://orcid.org/0000-00031087-0133. E-mail: julia.arrudagalvao@gmail.com

${ }^{3}$ Graduanda da Faculdade de Medicina na Universidade de Taubaté - UNITAU. ORCID iD: https://orcid.org/0000-00024676-7675. E-mail: assamara.freitas@gmail.com

${ }^{4}$ Doutora em Psicologia Ciência e Profissão pela Pontifícia Universidade Católica de Campinas - PUCCAMP. Pósdoutorado em Avaliação Psicológica pela Universidade São Francisco. Pós-Doutorado em Educação para Saúde na Universidade de Lisboa. Docente dos Cursos de Psicologia e Medicina da Universidade do Oeste Paulista. Docente e Coordenadora do Programa de Pós-Graduação - Mestrado e Doutorado em Educação - da Universidade do Oeste Paulista - UNOESTE. ORCID iD: https://orcid.org/0000-0003-3932-7580. E-mail: camelia@unoeste.br

${ }^{5}$ Mestre em Educação pela Universidade do Oeste Paulista - UNOESTE. Preceptora da Residência Médica em Psiquiatria do Hospital Regional de Presidente Prudente, Docente da Faculdade de Medicina da Universidade do Oeste Paulista - UNOESTE. ORCID iD: https://orcid.org/0000-0003-3932-7580. E-mail: dracelestebaptista@gmail.com

\section{RESUMO}

Pesquisas demonstram que os estudantes universitários estão mais vulneráveis à ocorrência de transtornos mentais do que a população em geral. Nesse sentido, o curso de Medicina, em especial, é considerado uma grande fonte de estresse. Este estudo teve como objetivo caracterizar as variáveis de adaptação acadêmica (pessoal, interpessoal, carreira, estudo e institucional) dos estudantes frente ao período de pandemia do COVID-19, bem como apresentar as estatísticas descritivas referentes às variáveis emocionais, físicas e comportamentais associadas à saúde mental (estresse, depressão e ansiedade). Participaram da pesquisa 513 estudantes de medicina, com idades variando entre 18 e 29 anos $(M=22,41$; $D P=2,49)$, oriundos de universidades públicas $(18,7 \% ; n=96)$ e privadas $(81,3 \% ; n=417)$. Foram utilizados três questionários para a coleta dos dados: o Questionário de Vivências Acadêmicas - versão reduzida (QVA-r); a Escala de Depressão, Ansiedade e Stress DASS-21 e um questionário sociodemográfico. A maior parte da amostra relatou prejuízos nos níveis de concentração $(79,5 \%)$ e no desempenho acadêmico $(59,8 \%)$ durante a pandemia, sendo que este contingente também não se sentiu apoiado pela universidade para lidar com as questões deste período. Em relação à atuação profissional, a maior parte dos estudantes $(53,6 \%)$ não se sente preparado para orientar condutas, bem como tem a percepção de que a pandemia interfere na relação com os pacientes na atenção básica $(93,8 \%)$. Em relação aos níveis de depressão, ansiedade e estresse, os alunos de primeiro e segundo ano apresentaram maiores médias quando comparado a alunos em estágios mais avançados. Este artigo contribuiu para identificar o impacto do ensino remoto na formação dos futuros médicos e, assim, fomentar a pesquisa e o estudo dessa nova adaptação frente à pandemia atual da COVID-19.

Palavras-chave: Infecções por Coronavírus; Pandemias; Comportamento Adaptativo; Estudantes de Medicina; Saúde Mental.

ACADEMIC ADAPTATION AND MENTAL HEALTH FOR MEDICINE STUDENTS AT COVID-19: EXPLORATORY STUDY IN BRAZIL

\section{ABSTRACT}


Research shows that university students are more vulnerable to the occurrence of mental disorders than the general population. In this sense, the medical course is considered a great source of stress. This study aimed to characterize the variables of academic adaptation (personal, interpersonal, career, study and institutional) of students facing the pandemic period of COVID-19, as well as to present descriptive statistics regarding emotional, physical and behavioral variables associated with mental health (stress, depression and anxiety). 513 medical students participated in the research, with ages varying between 18 and 29 years $(M=22.41 ; S D=2.49)$, coming from public $(18.7 \% ; n=96)$ and private universities $(81,3 \% ; n=$ 417). Three questionnaires were used for data collection: the Academic Experiences Questionnaire - short version (QVA-r); the Depression Scale, Anxiety and Stress DASS-21 and a sociodemographic questionnaire. It was observed that most of the sample reported losses in concentration levels (79.5\%) and academic performance (59.8\%) during the pandemic, and this contingent also did not feel supported by the university to deal with the issues of this period. Regarding professional performance, most students (53.6\%) do not feel prepared to guide conduct, as well as the perception that pandemic interferes in the relationship with patients in primary care (93.8\%). Regarding the levels of depression, anxiety and stress, the first and second year students had higher averages when compared to students in more advanced stages. This article contributed to identify the impact of remote education on the training of future doctors and, thus, encourage research and study of this new adaptation in the face of the current pandemic of COVID-19.

Keywords: Coronavirus Infections; Pandemics; Adaptative Behavior; Students, medical; Mental Health

\section{ADAPTACIÓN ACADÉMICA Y SALUD MENTAL DE ESTUDIANTES DE MÉDICO EN COVID19: ESTUDIO EXPLORATORIO EN BRASIL}

\section{RESUMEN}

La investigación muestra que los estudiantes universitarios son más vulnerables a la aparición de trastornos mentales que la población en general. En este sentido, el curso de medicina, en particular, se considera una gran fuente de estrés. Este estudio tuvo como objetivo caracterizar las variables de adaptación académica (personal, interpersonal, carrera, estudio e institucional) de los estudiantes que enfrentan el período pandémico del COVID-19, así como presentar estadísticas descriptivas sobre variables emocionales, físicas y conductuales asociadas a la salud mental. (estrés, depresión y ansiedad). Participaron en la investigación 513 estudiantes de medicina, con edades comprendidas entre 18 y 29 años ( $M=22,41$; $D T=2,49$ ), de universidades públicas $(18,7 \% ; n=96)$ y privadas $(81) .3 \% ; n=417)$. Se utilizaron tres cuestionarios para la recolección de datos: el Cuestionario de Experiencias Académicas - versión corta (QVA-r); la Escala de Depresión, Ansiedad y Estrés DASS-21 y un cuestionario sociodemográfico. La mayoría de la muestra reportó pérdidas en los niveles de concentración $(79,5 \%)$ y rendimiento académico $(59,8 \%)$ durante la pandemia, y este contingente tampoco se sintió apoyado por la universidad para atender los temas de este período. En cuanto a la práctica profesional, la mayoría de los estudiantes $(53,6 \%)$ no se sienten preparados para orientar comportamientos, además de tener la percepción de que la pandemia interfiere en la relación con los pacientes en atención primaria $(93,8 \%)$. En cuanto a los niveles de depresión, ansiedad y estrés, los estudiantes de primer y segundo año tuvieron promedios más altos en comparación con los estudiantes en etapas más avanzadas. Este artículo contribuyó a identificar el impacto de la enseñanza a distancia en la formación de los futuros médicos y, así, fomentar la investigación y el estudio de esta nueva adaptación ante la actual pandemia de COVID-19.

Palabras clave: Infecciones por coronavirus; Pandemias; Comportamiento adaptativo; Estudiantes de medicina; Salud mental.

\section{INTRODUÇÃO}

Em dezembro de 2019 iniciou-se na China um surto da doença causada pelo Coronavírus da síndrome respiratória aguda severa 2 (severe acute respiratory syndrome coronavirus 2 - Sars(CoV-2), que, dada sua alta capacidade de contaminação, se espalhou rapidamente pelo mundo, obrigando todos os governos a tomarem medidas drásticas para controle da disseminação, como a quarentena e o isolamento social (UDDIN, 2020) 
Em metanálise realizada com enfoque na prevalência de estresse e ansiedade entre a população em geral durante a pandemia de COVID-19, foi constatado que ele não só causa problemas de saúde física, como também resulta em distúrbios psicológicos. A faixa etária mais afetada é de 21 a 40 anos. Afigura-se como motivo a preocupação com as consequências futuras e com os desafios econômicos causados pela pandemia. Existe ainda uma associação entre os níveis de educação e os níveis de ansiedade e depressão, constatando-se maior prevalência de sintomas mentais entre pessoas com maior escolaridade. Esse fato decorre, provavelmente, do maior nível de autoconsciência desse grupo em relação à própria saúde (SALARI et al., 2020)

Nesse sentido, em abril de 2020 foi realizado, na Índia, um estudo para avaliação de depressão, ansiedade e estresse em 1000 entrevistados através da DASS-21, concluindo que a faixa etária entre 15 e 35 anos sofreu os maiores impactos, sendo que as mulheres são mais suscetíveis a desenvolverem sintomas de ansiedade e os homens são mais suscetíveis ao estresse e depressão (KAZMI et al., 2020).

Devido às medidas de controle de disseminação da COVID-19, as escolas e universidades foram fechadas $e$ as aulas presenciais suspensas afetando a rotina dos estudantes e gerando grandes preocupações relacionadas à saúde mental. Nesse momento de instabilidade, muitos transtornos mentais são deflagrados (SANTOS et al., 2020; ARAÚJO et al., 2020). Na intenção de trazer contribuições sobre o tema, realizaram uma pesquisa sobre adaptação acadêmica e o coping em universitários, a fim de compreender melhor se ocorreria correlação entre os construtos. Participaram do estudo 412 estudantes, utilizando o questionário de vivências acadêmicas- versão reduzida (QVA-r), escala modos de enfrentamento de problemas (EMEP) e um questionário sociodemográfico. Constatou-se que ter modos de encarar os problemas e um suporte social correlaciona-se de maneira positiva com a adaptação acadêmica. Foco na emoção ou pensamentos utópicos e práticas voltadas ao meio religioso correlacionaram-se negativamente com o meio acadêmico (DIAS et al., 2015).

Em paralelo, Malajovick et al. (2017) já vinham demonstrando que os estudantes universitários estão mais vulneráveis à ocorrência de transtornos mentais do que a população em geral. O curso de Medicina em especial é considerado uma grande fonte de estresse, pois contempla, além de todas as variáveis comuns aos outros cursos, a adaptação a um novo ambiente e a aquisição de identidade (SILVA; PEREIRA; MOURA, 2020). Os estudantes se deparam com uma carga horária maciça levando a esforços que dificultam o equilíbrio entre a vida pessoal e a acadêmica (SILVA; PEREIRA; MOURA, 2020).

Com o intuito de estimar a prevalência de depressão e sintomas depressivos em graduandos de medicina Rotenstein et al. (2016) extraíram de dados de prevalência de depressão ou sintomas depressivos de 167 estudos transversais ( $\mathrm{n}=116$ 628) e 16 estudos longitudinais ( $n=5728$ ), coletados em 43 países, e todos, exceto um estudo, utilizaram instrumentos de autorrelato. A prevalência global combinada de depressão ou sintomas depressivos foi de 27,2\%, e a análise dos 9 estudos que foram feitos de forma longitudinal e que observaram os sintomas antes e durante a faculdade de medicina demonstrou um aumento absoluto médio de sintomas de 13,5\%.

Já no Brasil, com o intuito de estimar a prevalência de distúrbios psiquiátricos nos estudantes de medicina da Universidade Federal do Rio Grande do Norte, Da Costa et al. (2020) constataram que em sua amostra, 66,3\% tinham estresse com predominância de sintomas psicológicos (42,3\%). Sintomas depressivos em diferentes graus se mostraram presentes em $28 \%$ dos estudantes. Em relação à sintomatologia ansiosa 66,3\% dos analisados apresentavam sinais de ansiedade em seu grau mínimo, e $33,7 \%$, sinais de ansiedade leve, moderada ou severa. Fica pois, claro que mesmo em períodos nos quais não estão instaladas situações de crise de alto impacto, os acadêmicos de medicina são muito afetados em relação à saúde mental.

No entanto, uma investigação realizada na China pretendeu mostrar como a pandemia da COVID- 19 repercutiu na saúde dos futuros médicos. Evidenciaram-se níveis mais elevados de ansiedade associados a fatores relacionados ao COVID-19 (CAO et al., 2020). Analogamente Zhai e Du (2020) mostraram que ocorreu um aumento da preocupação dos alunos em relação à sua educação, além do bem-estar de seus familiares ao voltarem para suas casas devido à suspensão das aulas presenciais. 
$\mathrm{Na}$ mesma direção, Rodrigues et al. (2020) sintetizaram o conteúdo de artigos e livros acerca do impacto da pandemia da COVID-19 na saúde mental e na educação superior dos estudantes de medicina. Essa análise concluiu que os estudantes de Medicina apresentam incertezas sobre o futuro de sua formação em decorrência das transformações ocasionadas pela pandemia, além de serem submetidos a uma carga emocional que deflagra danos à saúde mental. Existem ainda dúvidas sobre os reflexos desse contexto no período pós-COVID-19 e seu impacto na educação médica, assim como sobre a manutenção de medidas adotadas em tempos de crise.

A adaptação acadêmica é o processo vivenciado pelo estudante universitário diante das novas demandas do ensino superior. Dentre essas demandas pode-se mencionar o desempenho, o ajustamento às novas regras da instituição de ensino e o convívio social. Nessa etapa da vida do estudante comumente exige-se um questionamento pessoal em relação a vários domínios de sua existência, entre eles: vocacional, ético, acadêmico e social, o que promove o estabelecimento de novos padrões de funcionamento do indivíduo para a transposição desta fase de transição. A capacidade de adaptação às mudanças e às exigências que a universidade trará poderão interferir consideravelmente na forma como o estudante irá se adequar. Dessa forma, a adaptação à universidade, em especial ao curso médico, demandará suportar momentos instáveis ou que ameacem o estudante em algum grau. Uma rede de apoio à disposição do universitário pode ser um fator determinante para que este responda melhor diante de acontecimentos negativos, fornecendo suporte emocional e material conforme as demandas (SUEHIRO, 2004)

Pensando no contexto de sua inserção na vida acadêmica e compreendendo que a adaptação à universidade para muitos estudantes não ocorre de forma satisfatória, este estudo teve como objetivo caracterizar as variáveis de adaptação acadêmica (pessoal, interpessoal, carreira, estudo e institucional) dos estudantes frente ao período de pandemia da COVID-19 e apresentar as estatísticas descritivas referentes às variáveis emocionais, físicas e comportamentais associadas à saúde mental (estresse, depressão e ansiedade).

\section{DELINEAMENTO METODOLÓGICO}

Participaram do estudo 513 estudantes de medicina, com idades variando entre 18 e 29 anos $(M=22,41 ; D P=2,49)$, sendo $78,8 \%(n=$ 404) do gênero feminino, $21,1 \%(n=108)$ do gênero masculino e $0,2 \%(n=1)$ de gênero não declarado. Os participantes foram oriundos de universidades públicas $(18,7 \% ; n=96)$ e privadas $(81,3 \% ; n=417)$. No momento da pesquisa, que ocorreu entre os meses de julho a setembro de 2020, 88,7\% ( $n=455)$ informaram estarem participando de aulas online, $84 \%(n=431)$ estavam sendo submetidos a alguma forma de avaliação à distância e $90,3 \%$ ( $n=463)$ seguiam as regras de isolamento social.

Para coleta dos dados foi desenvolvido um protocolo na plataforma do Google Forms. Os participantes por meio de um link receberam as informações a respeito dos objetivos do estudo e o Termo de Consentimento Livre e Esclarecido (TCLE). A coleta dos dados ocorreu exclusivamente de forma online por meio de divulgação em snowball do link do protocolo do Google Forms via utilização das redes sociais online, bem como, contato via correio eletrônico (e-mail) com instituições de ensino superior brasileiras.

\section{INSTRUMENTOS}

Foram utilizados três questionários para a coleta dos dados. O Questionário de Vivências Acadêmicas - versão reduzida (QVA-r): Adaptado para o Brasil, que avalia cinco grandes áreas de vivências no âmbito universitário: pessoal, interpessoal, estudo, carreira e institucional (GRANADO et al., 2005). Também foi utilizado a Escala de Depressão, Ansiedade e Estresse DASS21 adaptada a versão Brasileira (LOVIBOND P.F; LOVIBOND S.H, 1995) .Trata-se de uma escala do tipo Likert de 4 pontos e 21 itens $(3=$ aplicada muito a mim, ou na maioria das vezes; 0 = não se aplica a mim de forma alguma) que se expressa em sentenças que descrevem estados emocionais negativos. Apresenta três subescalas (Depressão, Ansiedade e Estresse) e exibe boa consistência interna e validade convergente e discriminante. As pontuações em cada subescala variam de 0 a 21 pontos. A DASS tem demonstrado ser uma medida confiável e válida para a análise da saúde mental na população brasileira por apresentar qualidades psicométricas favoráveis e se mostrado como um instrumento adequado e relativamente simples de levantamento de sintomas de depressão, ansiedade e estresse no Brasil. (VIGNOLA; TUCCI, 2014). 
Foi aplicado também um questionário sociodemográfico composto por três conjuntos de indagações: a) Dados Pessoais dos discentes, tais como: gênero, faixa etária, estado civil, religião, filhos, etnia. b) Dados Profissionais: grau de escolaridade, semestre letivo, se tiveram aulas a distância, desempenho acadêmico e concentração, se obtiveram suporte da instituição de ensino, se se sentem preparados para assumir o papel de orientador de condutas técnicas às pessoas próximas ou quando abordados. c) Dados gerais: se reside sozinho, parentes ou conhecidos infectados com COVID19 , se cumpriram as regras do isolamento social. Todos assinaram Termo de Consentimento Livre e Esclarecido. O presente estudo foi aprovado pelo Comitê de Ética. (CAAE 31296220.8.0000.5515).

\section{ANÁLISE DE DADOS}

Os dados foram analisados por meio do software Statistical Package for the Social
Sciences (versão 23). Foram empregadas estatísticas descritivas para caracterização da amostra, do impacto da pandemia no âmbito acadêmico, na atuação e dos níveis de saúde mental. Na sequência foram adotadas estatísticas inferenciais para verificar as correlações entre os construtos por meio de Correlação de Pearson, potencial explicativo por meio de análise de regressão linear com método enter e possíveis diferenças de médias em função das variáveis demográficas por meio do teste $t$ de Student $e$ Análise de variância (ANOVA).

\section{RESULTADOS E DISCUSSÃO}

Inicialmente buscou-se verificar as respostas acerca das variáveis acadêmicas, da atuação dos estudantes durante a pandemia e do impacto nos níveis de saúde mental dos discentes de acordo com os critérios de classificação do Questionário Sociodemográfico. Os resultados são apresentados na Tabela 1. 
Tabela 1. Impactos da Pandemia no desempenho acadêmico

\begin{tabular}{|c|c|c|c|c|}
\hline Âmbito & Variável & Categorias & $f$ & $\%$ \\
\hline \multirow[t]{8}{*}{ ACADÊMICO } & \multirow{3}{*}{$\begin{array}{l}\text { Desempenho acadêmico durante } 0 \text { isolamento } \\
\text { social }\end{array}$} & Melhorou & 102 & 19,9 \\
\hline & & Piorou & 307 & 59,8 \\
\hline & & Não se modificou & 104 & 20,3 \\
\hline & \multirow[t]{3}{*}{ Concentração durante o isolamento social } & Melhorou & 51 & 9,9 \\
\hline & & Piorou & 408 & 79,5 \\
\hline & & Não se modificou & 54 & 10,5 \\
\hline & \multirow[t]{2}{*}{ Se sente apoiado pela universidade na pandemia } & Sim & 114 & 22,2 \\
\hline & & Não & 399 & 77,8 \\
\hline \multirow{8}{*}{$\begin{array}{l}\text { ATUAÇÃO } \\
\text { PANDEMIA }\end{array}$} & \multirow{3}{*}{$\begin{array}{l}\text { Se sentiu preparado para assumir o papel de } \\
\text { orientador de condutas técnicas }\end{array}$} & Sim & 221 & 43,1 \\
\hline & & Não & 275 & 53,6 \\
\hline & & Não respondeu & 17 & 3,3 \\
\hline & \multirow{3}{*}{$\begin{array}{l}\text { Foi orientado para esclarecer e encaminhar pessoas } \\
\text { suspeitas por: }\end{array}$} & Faculdade & 116 & 22,6 \\
\hline & & $\begin{array}{l}\text { Mídia } \\
\text { especializada }\end{array}$ & 229 & 44,6 \\
\hline & & Mídia leiga & 168 & 32,7 \\
\hline & \multirow{2}{*}{$\begin{array}{l}\text { A pandemia pode interferir na relação estudante- } \\
\text { paciente na atenção básica }\end{array}$} & Sim & 481 & 93,8 \\
\hline & & Não & 32 & 6,2 \\
\hline \multirow[t]{3}{*}{ SAÚDE MENTAL } & \multirow[t]{3}{*}{ O isolamento impactou nas relações interpessoais } & Sim & 426 & 83,0 \\
\hline & & Não & 71 & 13,8 \\
\hline & & $\begin{array}{l}\text { Não cumpri o } \\
\text { isolamento }\end{array}$ & 16 & 3,1 \\
\hline
\end{tabular}

Fonte: O autor 2021.

Observa-se que a maior parte da amostra relatou prejuízos nos níveis de concentração (79,5\%) e no desempenho acadêmico $(59,8 \%)$ durante a pandemia, sendo que este contingente também não se sente apoiado pela universidade para lidar com as questões deste período. Em relação à atuação profissional, a maior parte dos estudantes $(53,6 \%)$ não se sente preparada para orientar condutas, bem como tem a percepção de que a pandemia interfere na relação com os pacientes na atenção básica $(93,8 \%)$. Na sequência foram feitas comparações de média entre os instrumentos da DASS 21 (Escala de Depressão, Ansiedade e Estresse) e QVA-r (Questionário de Vivências Acadêmicas - versão reduzida) por meio do teste $t$ de Student em função do apoio recebido por parte da universidade (Tabela 2). 
Tabela 2. Médias nas dimensões de Adaptação Acadêmica e Variáveis de Saúde Mental em função do apoio recebido pela Universidade.

\begin{tabular}{|c|c|c|c|c|c|}
\hline $\begin{array}{c}\text { Dimensões de } \\
\text { Adaptação Acadêmica }\end{array}$ & $\mathrm{T}$ & $d f$ & $\begin{array}{l}\text { Se sente esclarecido e apoiado pela } \\
\text { faculdade neste momento de pandemia? }\end{array}$ & $M$ & $\mathrm{DP}$ \\
\hline \multirow[t]{2}{*}{ Carreira } & $1,914^{*}$ & 511 & Sim & 51,11 & 6,39 \\
\hline & & & Não & 49,71 & 7,04 \\
\hline \multirow[t]{2}{*}{ Pessoal } & $6,312^{* *}$ & 511 & Sim & 43,28 & 11,96 \\
\hline & & & Não & 35,70 & 11,12 \\
\hline \multirow[t]{2}{*}{ Interpessoal } & $2,578^{*}$ & 511 & Sim & 47,68 & 8,07 \\
\hline & & & Não & 45,10 & 9,81 \\
\hline \multirow[t]{2}{*}{ Estudo } & $4,220^{* *}$ & 511 & Sim & 32,46 & 5,84 \\
\hline & & & Não & 29,68 & 6,30 \\
\hline \multirow[t]{2}{*}{ Institucional } & $6,312^{* *}$ & 511 & Sim & 30,46 & 3,92 \\
\hline & & & Não & 27,46 & 4,63 \\
\hline $\begin{array}{c}\text { Variáveis de Saúde } \\
\text { Mental }\end{array}$ & $\mathrm{T}$ & $d f$ & $\begin{array}{l}\text { Se sente esclarecido e apoiado pela } \\
\text { faculdade neste momento de pandemia? }\end{array}$ & $M$ & $\mathrm{DP}$ \\
\hline \multirow[t]{2}{*}{ Depressão } & $4,008^{* *}$ & 510 & Sim & 6,10 & 5,05 \\
\hline & & & Não & 8,65 & 6,22 \\
\hline \multirow[t]{2}{*}{ Ansiedade } & $3,353^{* *}$ & 510 & Sim & 5,82 & 5,27 \\
\hline & & & Não & 7,95 & 6,14 \\
\hline \multirow[t]{2}{*}{ Estresse } & $3,703^{* *}$ & 510 & Sim & 10,04 & 5,85 \\
\hline & & & Não & 12,38 & 5,96 \\
\hline
\end{tabular}

Fonte: $\mathrm{O}$ autor 2021.

Nota: $p<0,05 ; p<0,001$

Em relação à adaptação acadêmica, os alunos que se sentem apoiados e esclarecidos pela universidade apresentaram maiores médias nos fatores do Questionário de Adaptação Acadêmica (QVA-r). Essas médias foram maiores nas seguintes dimensões: carreira, pessoal, interpessoal e estudo indicando que esses participantes aparentam perspectivas positivas quanto ao futuro profissional, estão satisfeitos com o curso e sentem-se mais competentes para realização das tarefas acadêmicas (Dimensão
Carreira). As médias representativas da Dimensão Pessoal indicam que os estudantes percebem-se mais otimistas, autoconfiantes e com maior facilidade para tomada de decisões.

Os alunos que afirmaram estar sendo esclarecidos e apoiados pela faculdade no momento da pandemia, informaram ainda que possuem vínculos significativos de amizade e contam com o suporte de professores (Dimensão Interpessoal). Igualmente, revelaram que conseguiram manter rotinas de estudo 
organizadas, planejamento de tempo e fazerem uso de recursos de pregação para as provas.

Em contrapartida, aqueles com menor percepção de esclarecimento e apoio das universidades apresentaram maiores médias de depressão, ansiedade e estresse. O estresse é uma reação natural que o indivíduo apresenta em situações de perigo e que o coloca em estado de alerta. Nesse sentido, essa tensão derivada do estado de alerta pode gerar um sentimento desagradável e de medo caracterizado pela ansiedade. Ao passo que a depressão é uma doença psiquiátrica descrita por uma tristeza profunda, anedonia e oscilações de humor.

Em seguida foi feita a comparação de médias em função do momento de graduação dos estudantes. Para isso, cada ano foi composto por dois semestres. Os resultados significativos são apresentados na Tabela 3. 
Tabela 3. Pontuações nas Dimensões de Adaptação Acadêmica e Variáveis Estresse, Depressão e Ansiedade em função do Ano de Graduação

\begin{tabular}{|c|c|c|c|c|c|}
\hline Fator & gl & $\mathrm{F}$ & Ano & \multicolumn{2}{|c|}{ Subconjuntos } \\
\hline \multicolumn{6}{|l|}{ Dimensões de Adaptação Acadêmica } \\
\hline \multirow[t]{6}{*}{ Interpessoal } & 5 & $2,562^{*}$ & 2 & 43,82 & \\
\hline & & & 1 & 44,12 & \\
\hline & & & 3 & 44,80 & \\
\hline & & & 6 & 46,34 & \\
\hline & & & 4 & 47,12 & \\
\hline & & & 5 & 47,54 & \\
\hline \multirow[t]{6}{*}{ Depressão } & 5 & $2,910^{*}$ & 6 & 6,60 & \\
\hline & & & 4 & 7,11 & 7,11 \\
\hline & & & 5 & 7,39 & 7,39 \\
\hline & & & 3 & 8,52 & 8,52 \\
\hline & & & 1 & 9,22 & 9,22 \\
\hline & & & 2 & & 9,35 \\
\hline \multirow[t]{6}{*}{ Ansiedade } & 5 & $2,562^{*}$ & 6 & 5,92 & \\
\hline & & & 4 & 6,45 & 6,45 \\
\hline & & & 3 & 7,28 & 7,28 \\
\hline & & & 5 & 7,65 & 7,65 \\
\hline & & & 1 & 8,52 & 8,52 \\
\hline & & & 2 & & 8,66 \\
\hline \multirow[t]{6}{*}{ Estresse } & 5 & $2,356^{*}$ & 6 & 10,21 & \\
\hline & & & 4 & 11,07 & 11,07 \\
\hline & & & 5 & 11,76 & 11,76 \\
\hline & & & 3 & 11,81 & 11,81 \\
\hline & & & 2 & 12,73 & 12,73 \\
\hline & & & 1 & & 13,13 \\
\hline
\end{tabular}


Os alunos de quinto ano apresentaram maiores médias de adaptação interpessoal, que se refere às relações com colegas e à habilidade de se relacionar em situações de maior intimidade e de amizade quando comparados com os alunos dos demais anos. Em relação aos níveis de depressão, ansiedade e estresse, os alunos de primeiro e segundo ano apresentaram maiores médias e diferenciam-se dos alunos de último ano que apresentaram melhores níveis de saúde mental.

Uma pesquisa realizada em 2011 pela Associação Nacional dos Dirigentes das Instituições Federais de Ensino Superior (Andifes) traçou o perfil dos estudantes das universidades federais e, para isso, uma das áreas mapeadas foi a de recorrência de transtornos mentais nessa parcela específica da população. A conclusão foi de que metade dos universitários brasileiros havia vivenciado uma crise emocional no ano anterior e que o transtorno que mais afetava o desempenho dos alunos era o de ansiedade, com o qual $70 \%$ dos entrevistados se identificavam (FONAPRACE, 2018).

Na pandemia da COVID-19 tal realidade, já causadora de sofrimentos exigiu ainda mais dos universitários. Nessa nova realidade, o uso de novas ferramentas como, por exemplo, as aulas remotas colaboraram ainda mais para a intensificação do estresse e ansiedade dos estudantes. Pode-se inferir tal afirmativa através dos achados obtidos por meio do questionário sociodemográfico no âmbito acadêmico. Esses demonstraram que, durante a pandemia, a maior parte dos alunos obteve piora na sua concentração e desempenho acadêmico, além de não se sentirem apoiados pelas universidades para lidar com os problemas e mudanças advindos desse período.

Achados semelhantes foram também encontrados por Soares et al. (2018), evidenciando que habilidades socialmente aceitáveis para a vida universitária estão relacionadas não somente a variáveis pessoais, mas também a questões curriculares, institucionais e externas ao indivíduo. Nesse prisma, o impacto da quarentena no bem-estar subjetivo e nos comportamentos de aprendizagem de estudantes de medicina foi investigado em pesquisa constatando que um quarto desses estudantes se sentiu desanimado durante esse período. $\mathrm{O}$ que, segundo Meo et al. (2020) poderia ser um indicativo de comprometimento nos perfis psicológicos e na aprendizagem desses alunos a longo prazo. Portanto, vivenciar um período de pandemia repercute substancialmente na vida dos indivíduos, levando-os obrigatoriamente a mudanças em seus padrões de relacionamento interpessoal e mobilizando emoções e comportamentos inesperados.

Embasando o estudo, cabe salientar uma revisão de literatura acadêmica realizada para identificar as estratégias pedagógicas para a educação médica implementadas durante a pandemia da Covid-19 em diferentes países do mundo e após análise de 27 artigos concluiu-se que apesar de a tecnologia ser um recurso pedagógico fundamental na pandemia, a maioria dos estudos não aponta as fragilidades, somente as defende de forma acrítica e sem o embasamento teórico necessário para que assim seja garantida uma melhor prática da educação médica (SANTOS et al., 2020).

Dessa forma, evidencia-se que estudantes do curso médico foram expostos a exigências que transcenderam às expectativas do seu já característico período acadêmico. Foram surpreendidos pelas exigências legais embasadas em informações da OMS (Organização Mundial de Saúde) obrigando as escolas a alterar seus métodos de ensino. Sem tempo hábil para se realinhar a este chamado, cada escola improvisou as mudanças utilizando as tecnologias disponíveis em cada instituição. Esta realidade impôs ao estudante de medicina mais uma sobrecarga.

$\mathrm{Na}$ mesma direção, Carrascosa et al. (2020) avaliaram o impacto da COVID-19 em instituições de ensino médico públicas e privadas do Brasil. Como resultado, observou-se que em relação à atuação profissional a maior parte dos estudantes $(53,6 \%)$ não se sentiu preparada para orientar condutas, visto que mais da metade dos alunos não recebeu nenhum conteúdo teórico sobre a COVID-19 $(58,4 \%)$, nem treinamento sobre o uso de $\operatorname{EPI}(57,1 \%)$, bem como tiveram a percepção de que a pandemia interfere na relação com os pacientes na atenção básica $(93,8 \%)$. Esses achados demonstraram que apenas $10,7 \%$ dos entrevistados se consideravam seguros para atender no pronto-socorro durante o período da pandemia, e $80,8 \%$ tinham medo de se contaminar com o vírus. Condizem assim, com o presente estudo, no qual a maioria dos estudantes $(53,6 \%)$ não se sente preparada para orientar condutas, bem como tem a percepção 
de que a pandemia interfere na relação com os pacientes na atenção básica (93,8\%).

Dados analisados na Tabela 3, que relaciona as pontuações de adaptação acadêmica e variáveis de saúde mental baseadas no ano de graduação, demonstraram que os alunos que se encontravam em períodos mais avançados do curso de medicina apresentaram maior média de adaptação interpessoal, que se refere às relações com colegas e habilidade de se relacionar em situações com maior intimidade e amizades. Uma maior média também foi encontrada para o quesito de saúde mental. Os graduandos são beneficiados pelo acúmulo das experiências vividas, e isso os ajuda no confronto das fantasias vs. realidade do dia a dia do ensino superior, bem como pelo aprimoramento de suas habilidades de estudo (IGUE; BARIANI; MILANESI, 2008).

\section{CONCLUSÕES}

O período de inserção no Ensino Superior é determinante nas relações interpessoais, na saúde mental, na aprendizagem e desempenho acadêmico e, principalmente na continuidade ou não da graduação. O suporte afetivo, financeiro e institucional, são descritos como preditores de adaptação ao contexto universitário.

O presente estudo evidenciou grande sofrimento e sobrecarga emocional em estudantes de cursos de Medicina no período da Pandemia COVID-19. Fica claro que as instituições de ensino não conseguiram mitigar as angústias dos estudantes. $O$ ensino remoto, apesar de garantir a apresentação do conteúdo curricular, não atendeu às expectativas dos estudantes que sentiram que seu desempenho estava aquém de seus anseios, não garantindo segurança quanto ao seu aprendizado.

Ademais, ações de acolhimento, amparo e humanização provindas das instituições de ensino superior apresentaram resultado significativo na adaptação acadêmica e saúde mental dos alunos de medicina, diminuindo sua vulnerabilidade, caracterizando-se assim como um importante fator protetivo para a população estudada.

Este artigo contribuiu para identificar o impacto do ensino remoto na formação dos futuros médicos; contudo, o fato de haver um desequilíbrio acentuado nas variáveis de gênero e nas universidades de onde são oriundos são limitações. Comparações entre estudantes de regiões distintas do Brasil também poderiam trazer informações pertinentes. Novos estudos poderiam apresentar resultados interessantes, comparando, por exemplo, o desempenho dos estudantes dentro deste modelo de ensino remoto entre escolas médicas que ainda possam estar aplicando uma metodologia mais próxima da tradicional e as escolas com metodologias ativas plenamente implementadas.

\section{REFERÊNCIAS}

ARAÚJO, F. et al. Impact Of Sars-Cov-2 And Its Reverberation In Global Higher Education And Mental Health. Psychiatry research., v. 288, n. 112977. 2020.

https://doi.org/10.1016/j.psychres.2020.112977

CAO, W. et al. The psychological impact of the COVID-19 epidemic on college students in China. Psychiatry research., v. 287, n. 112934, 2020.

https://doi.org/10.1016/i.psychres.2020.112934

CARRASCOSA, M. et al. Medical Interns and COVID-19: results of national research. Rev Bras Educ Med., v. 66, $n$ 6, p. 812-817, 2020. https://doi.org/10.1590/1806-9282.66.6.812

DA COSTA, D. S. et al. Sintomas de depressão, ansiedade e estresse em estudantes de Medicina e estratégias institucionais de enfrentamento. Rev Bras Educ Med., v. 44, n. 1, p. 1-10, 2020. https://doi.org/10.1590/1981-5271v44.1$\underline{20190069}$

DIAS, J. C. R. et al. Escala de estresse percebido aplicada a estudantes universitários: estudo de validação. Psychology, Community \& Health, v. 4, n. 1, p. 1-13, mar., 2015.

GRANADO, J. I. et al. Integração acadêmica de estudantes universitários: contributos para a adaptação e validação do QVA-r no Brasil. Psicologia e Educação. v. IV, n, 2, p. 3541, dez., 2005.

IGUE, É. A.; BARIANI, I. C. D.; MILANESI, P. V. B. Vivência acadêmica e expectativas de universitários ingressantes e concluintes. PsicoUSF., v. 13, n. 2, p. 155-64, dez., 2008.

https://doi.org/10.1590/S1413$\underline{82712008000200003}$

KAZMI, S. S. H. COVID-19 e Lockdwon: a study on the mental health. SSRN, abr., 2020.

https://doi.org/10.2139/ssrn.3577515 
LOVIBOND, P.F.; LOVIBOND S.H. A estrutura dos estados emocionais negativos: Comparação das Escalas de Depressão, Ansiedade e Estresse (DASS) com os Inventários de Depressão e Ansiedade de Beck. Behavior Research and Therapy., v. 33, n. 3, p. 335-343, 1955.

MALAJOVICH, N. et al. A juventude universitária na contemporaneidade: a construção de um serviço de atenção em saúde mental para estudantes. Mental, v. 11, n. 21, p. 22, 2017.

MEO, S.A. et al. COVID-19 Pandemic: impact of quarantine on medical students' mental wellbeing and learning behaviors. Pak J Med Sci., v. 36 , n. 4, p. 1-6, 2020. DOI:

https://doi.org/10.12669/pjms.36.COVID19$\underline{\mathrm{S} .2809}$

OBSERVATÓRIO DO FÓRUM NACIONAL DE PRÓREITORES DE ASSUNTOS ESTUDANTIS (FONAPRACE). V Pesquisa Nacional de Perfil Socioeconômico e Cultural dos (as) Graduandos (as) das IFES. Uberlândia, MG: Universidade Federal de Uberlândia, 2018. Disponível em: https://www.andifes.org.br/wpcontent/uploads/2019/05/V-Pesquisa-do-PerfilSocioecon\%C3\%B4mico-dos-Estudantes-deGradua\%C3\%A7\%C3\%A3o-das-UniversidadesFederais-2.pdf. Acesso em: 11 dez de 2020.

RODRIGUES, B. B. et al. Aprendendo com o imprevisível: saúde mental dos universitários e Educação Médica na pandemia de Covid-19. Rev Bras Educ Médica. v. 44, n. 1, p. 149, 2020. https://doi.org/10.1590/1981-5271v44.supl.1$\underline{20200404}$

ROTENSTEIN, L. S. et al. Prevalence of depression, depressive symptoms, and suicidal ideation among medical students: a systematic review and meta-analysis. JAMA, v. 316, n. 21, p. 2214-2236, 2016.

https://doi.org/10.1001/jama.2016.17324

SALARI, N.; et al. Prevalence of stress, anxiety, depression among the general population during the COVID-19 pandemic: a systematic review and meta-analysis. Glob Health, v. 16, n. 1, p. 57, dez., 2020. https://doi.org/10.1186/s12992-02000589-w

SANTOS, B. M. et al. Educação médica durante a pandemia da Covid-19: uma revisão de escopo.
Rev Bras Educ Médica. v. 44, n. 1, p. 139, 2020. https://doi.org/10.1590/1981-5271v44.supl.1$\underline{20200383}$

SILVA, R. C.; PEREIRA A. A.; MOURA, E. P. Qualidade de Vida e Transtornos Menores dos Estudantes de Medicina do Centro Universitário de Caratinga (UNEC). Rev Bras Educ Médica, v. 44, n. 2, p. 64, 2020.

https://doi.org/10.1590/1981-5271v44.2$\underline{20190179}$

SOARES, A. B. et al. Vivências, habilidades sociais e comportamentos sociais de universitários. Psicol. Teor. e Pesqui. v. 34, n. 34311, 2018. https://doi.org/10.1590/0102.3772e34311

SUEHIRO, Adriana Cristina Boulhoça. Estudante universitário: características e experiências de formação. Psico-USF (Impr.), Itatiba, v. 9, n. 1, p. 105-106, june., 2004.

https://doi.org/10.1590/S1413-

$\underline{82712004000100013}$

UDDIN, M. et al. SARS-CoV-2/COVID-19: genômica viral, epidemiologia, vacinas e intervenções terapêuticas. Vírus. v. 12, n. 5, p. 526, 2020. https://doi.org/10.3390/v12050526

VIGNOLA, R. C.; TUCCI, A. M. Adaptation and validation of the depression, anxity and stress scale (DASS) to Brazilian Portuguese. J Affect Disord, v. 155, p. 104-109. 2014.

https://doi.org/10.1016/j.jad.2013.10.031

ZHAI, Y.; DU, X. Abordando a saúde mental nas unicersidades em meio à pandemia de COVID-19. Psychiatry Res., v. 288, 2020.

https://doi.org/10.1016/j.psychres.2020.113003 